\title{
Antibiotic Sensitivity Patterns of Urine and Biofilms in Patients with Indwelling Urinary Catheter in Denden Hospital, Asmara, Eritrea
}

\author{
Lia Alem ${ }^{1}$, Salih Mohammed ${ }^{1}$, Mohammed Elfatih Humida ${ }^{2}$, Berzelin Adugna ${ }^{1}$, \\ Feven G. Medhin ${ }^{1}$, Temesgen Weldu ${ }^{1}$
}

${ }^{1}$ Microbiology, National Health Laboratory, Asmara, Eritrea

${ }^{2}$ Microbiology, Orotta School of Medicine and Health Sciences, Asmara, Eritrea

Email: *Liawitgeneta555@gmail.com

How to cite this paper: Alem, L., Mohammed, S., Humida, M.E., Adugna, B., Medhin, F.G. and Weldu, T. (2019) Antibiotic Sensitivity Patterns of Urine and Biofilms in Patients with Indwelling Urinary Catheter in Denden Hospital, Asmara, Eritrea. Advances in Microbiology, 9, 131-138. https://doi.org/10.4236/aim.2019.92010

Received: December 29, 2018

Accepted: January 31, 2019

Published: February 3, 2019

Copyright $\odot 2019$ by author(s) and Scientific Research Publishing Inc. This work is licensed under the Creative Commons Attribution International License (CC BY 4.0).

http://creativecommons.org/licenses/by/4.0/

\begin{abstract}
The intricate infections leading to long-term morbidity of catheterized patients are due to the presence of a covering and blocking the lumen of urinary catheters by biofilms which have increased ability of resistance to host immune system and antibiotic treatment. The biofilm mode of growth is a basic survival strategy implemented by bacteria in a wide range of settings such as environmental, industrial and clinical aquatic settings. Bacterial growth on the inner surface of the catheter with biofilm formation is frequent and may occur within days of catheter placement. This study investigated the formation of biofilm inside catheter lumen of patients from Denden hospital, Asmara, Eritrea. And also, it assessed the antimicrobial sensitivity pattern of biofilm isolates and compared it with urine isolates. Resistance to antibiotics was observed in biofilm isolates more than urine isolates. E. coli was the most frequently isolated organism in both biofilm and urine samples.
\end{abstract}

\section{Keywords}

Biofilm, Catheter-Associated Urinary Tract Infection, Eritrea

\section{Introduction}

Denden Hospital was founded in 1976-77 in Port Sudan to provide care to the heavily damaged fighters in various battles against the Ethiopian forces. After independence the hospital moved to Asmara, Eritrea giving service to the patriots of the war for independence as well as to the late youngsters who participated in securing independence. The hospital has around 30 beds for inpatient service and has registered regular users of over 400 people in the outpatient de- 
partment. Out of all the patients over 100 have urine control problem, therefore they use catheter to relieve urinary retention and urinary incontinence.

Individuals requiring an indwelling catheter are predisposed to the development of catheter associated urinary tract infections (CAUTIs) mostly due to two reasons, the presence of an indwelling catheter device and presence of potentially pathogenic multidrug-resistant organisms [1]. Despite the immediate threat of infection from these potent opportunistic nosocomial multiresistant strains, most catheter-associated bacteriuria or the presence of bacteria in the urine are asymptomatic [2]. However, when an incident of CAUTI becomes symptomatic, the resulting sequela can range from mild diseases like fever, cystitis and urethritis to severe: acute pyelonephritis, renal scarring, and bacteremia [2] [3].

Systemic antibiotics frequently fail to treat biofilm-associated infections since biofilms afford the bacteria a lot of survival advantages by providing genetic exchange between the organisms thereby allowing them to develop resistance against host immune response and to antibiotics also sessile bacteria in the biofilms are usually less susceptible to killing by antimicrobial agents than their rapidly growing planktonic counterpart cells [4]. The risk of urinary tract infection is related to the duration of catheterization. Long-term catheterization leads to colonization by extensive biofilms, which can have profound effect on the health of patients [5] [6].

\section{Methodology}

This is a prospective cross-sectional study done in the microbiology department of the national health laboratory, Asmara, Eritrea during the period of 1st January, 2018 to 31st October, 2018. Patients from denden hospital, Asmara, who have undergone indwelling catheterization for urinary retaining and some for urinary incontinence has developed biofilm in the inner surface of catheters which could be seen by naked eye was included in this study. Patient's clinical presentation and duration of catheterization was noted in data sheet. Urine sample was collected in sterile, wide mouth container through suprapubic puncture. A cross section of indwelling catheter containing biofilm seen on naked eye was our second sample. A modified standardized semi-quantitative technique using calibrated bacteriological loop of urine was performed to transfer the $10 \mathrm{ul}$ of sample on Nutrient agar, Mackonkey agar and Bile esculin agar. After allowing the urine to be absorbed into the agar, the plates were then inverted and incubated aerobically at $37^{\circ} \mathrm{C}$ for 24 hours. Bacterial colonies greater than 10 were considered as significant and were aseptically isolated. Antimicrobial sensitivity test was carried out by disc diffusion technique using Muller Hinton Agar. The Antibiotics used for susceptibility testing of gram negative bacilli were amikacin, ceftriaxone, nitrofurantoin, ceftazidime, tetracycline, ciprofloxacin, cotrimoxazole, cephalexin, nalidixic acid, ampicillin, chloramphenicol and gentamycin. And for gram positive cocci we used tetracycline, oxacillin, vancomycin, erythromycin, rifampin, clindamycin, Nitrofurantoin, chloramphenicol, ciproflox- 
acin, gentamycin For collecting the data for results of urine and biofilm culture, microbiology laboratory's request form was used to write down every growth seen from the urine and biofilm culture. And data was entered and analyzed in WHONET5.5 software.

\section{Result}

From 50 urine samples processed, 74 uropathogens were isolated, $21.6 \%$ of the isolates were $E$. coli which accounted for majority of the isolates followed by $\mathrm{Ci}$ trobacter diversus which accounted for $9.5 \%$ of the total uropathogens isolated in urine sample (see Figure 1), Morganella morgani was found in 9.5\% samples, Proteus mirabilis 8.1\%, Klebsiella pneumonia 6.8\% and Enterococcus faecalis in same as Klebsiella $6.8 \%$ of the samples.

Out of 50 biofilm samples collected 102 uropathogens were isolated, which suggests that there was multiple uropathogen isolation from single sample. As we can see in Figure 2 the most frequently isolated microorganism was $E$. coli (19.6\%), followed by Enterococcus faecalis (16.7\%), Proteus mirabilis (15.7\%), Morganella morgani (4.9\%), Citrobacter diversus and Providencia retgeri same incidence $3.92 \%$ both.

\section{Antibiotic sensitivity pattern of organisms isolated in urine}

As we can see from Table 1 the antibiotic sensitivity patterns of organisms isolated in urine showed, a sensitivity pattern of E. coli against amikacin, ampicillin, cephalexin, ceftazidime, ceftriaxone, chloramphenicol, ciprofloxacin, co-trimaxazole, gentamycin, nalidixic acid, nitrofurantoin, and tetracycline was $87.5 \%, 6.25 \%$, 25\%, 31.25\%, 43.75\%, 87.5\%, 25\%, 31.25\%, 56.25\%, 18.75\%, 81.25\%, $31.25 \%$ respectively.

$\mathrm{R}=$ Rifampin, $\mathrm{V}=$ Vancomycin (Enterococcus faecalis only six drugs are used since it is gram positive streptococcus); Amikacin is not prescribed in Denden hospital so the organisms came to be sensitive to it, since the organisms are not introduced to that drug we excluded it from antimicrobial comparison. To make

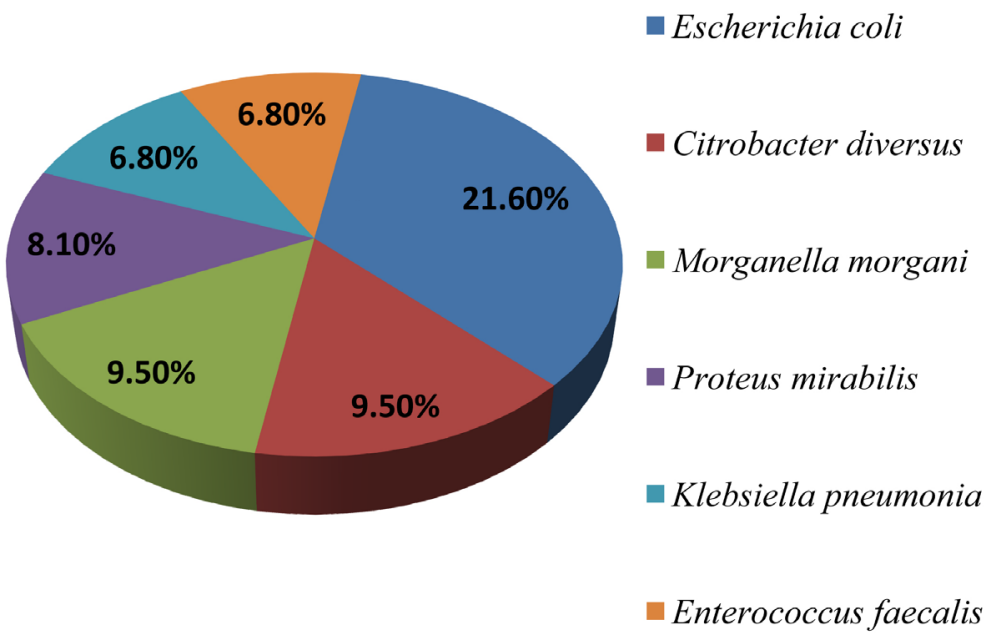

Figure 1. Percentage distribution of six most isolated uropathogens from urine sample. 
Table 1. Antibiotic sensitivity pattern of organisms isolated in urine.

\begin{tabular}{|c|c|c|c|c|c|c|c|c|c|c|c|c|c|}
\hline \multirow[t]{2}{*}{ Organism Isolated } & \multicolumn{12}{|c|}{ Sensitive } & \multirow{2}{*}{$\begin{array}{c}\text { Resistant to all } \\
0 \text { (without } \\
\text { Amikacin 5) }\end{array}$} \\
\hline & Ak & Am & $\mathrm{Clx}$ & $\mathrm{Caz}$ & Cro & Chl & Cip & Sxt & $\mathrm{Gm}$ & $\mathrm{Nal}$ & Nif & $\mathrm{Te}$ & \\
\hline E. coli & $87.5 \%$ & $6.25 \%$ & $25 \%$ & $31.25 \%$ & $43.75 \%$ & $87.5 \%$ & $25 \%$ & $31.25 \%$ & $56.25 \%$ & $18.75 \%$ & $81.25 \%$ & $31.25 \%$ & \\
\hline Citrobacter diversus & $85.7 \%$ & $0 \%$ & $28.6 \%$ & $28.6 \%$ & $57.1 \%$ & $28.57 \%$ & $42.8 \%$ & $28.6 \%$ & $28.6 \%$ & $14.2 \%$ & $57.1 \%$ & $14.2 \%$ & \\
\hline Proteus mirabilis & $100 \%$ & $0 \%$ & $16.7 \%$ & $16.7 \%$ & $16.7 \%$ & $16.7 \%$ & $16.7 \%$ & $0 \%$ & $16.7 \%$ & $0 \%$ & $16.7 \%$ & $16.7 \%$ & \\
\hline Providencia retgeri & $100 \%$ & $0 \%$ & $20 \%$ & $80 \%$ & $60 \%$ & $60 \%$ & $80 \%$ & $0 \%$ & $20 \%$ & $20 \%$ & $0 \%$ & $0 \%$ & \\
\hline Enterococcus faecalis & - & $100 \%$ & $0 \%$ & $\mathrm{R}-20 \%$ & $\mathrm{~V}-0 \%$ & - & - & - & $20 \%$ & - & - & $0 \%$ & \\
\hline Klebsiella pneumonia & $80 \%$ & $0 \%$ & $0 \%$ & $0 \%$ & $0 \%$ & $80 \%$ & $0 \%$ & $0 \%$ & $0 \%$ & $0 \%$ & $80 \%$ & $20 \%$ & \\
\hline
\end{tabular}

\section{BIOFILM}

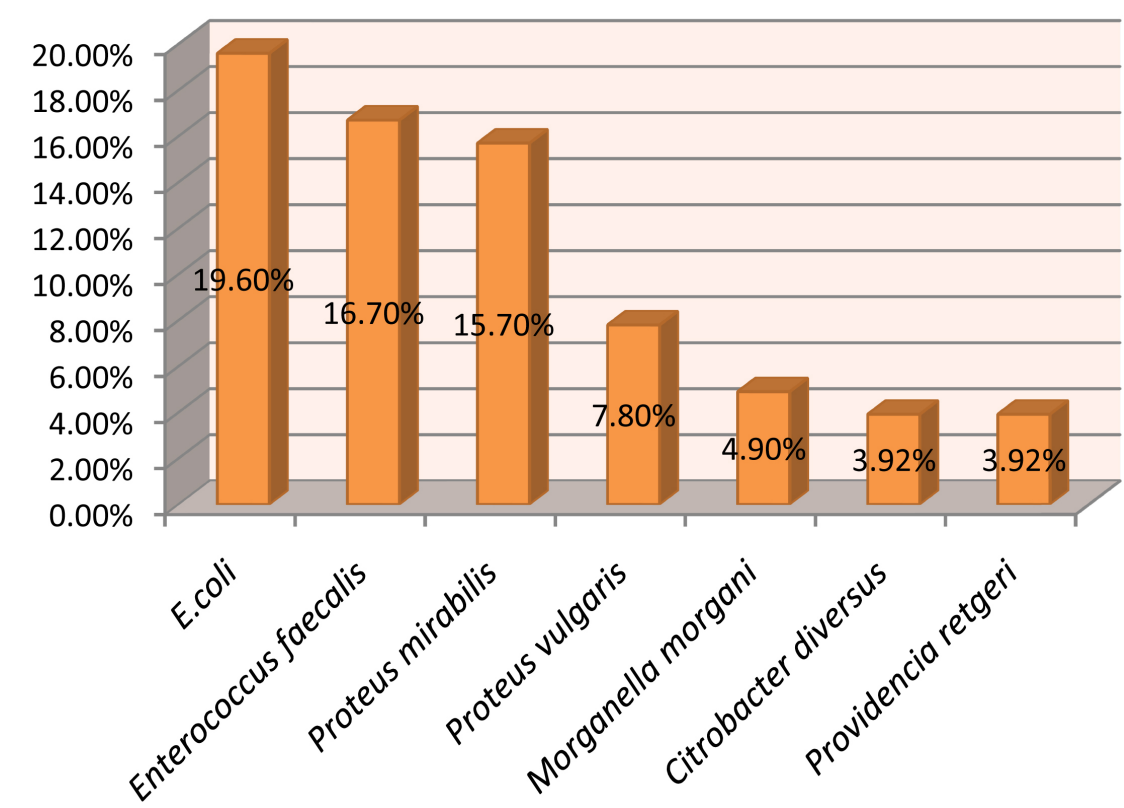

Figure 2. Percentage distribution of seven most isolated uropathogens in biofilm.

it clear, since amikacin is not prescribed for denden hospital catheterized patients due to some reasons, five of the isolates were resistant to the 11 antimicrobials used except to amikacin.

As we can see from Table 2, the antibiotic sensitivity pattern of organisms isolated in biofilm showed, a sensitivity pattern of $E$. coli against amikacin, ampicillin, cephalexin, ceftazidime, ceftriaxone, chloramphenicol, ciprofloxacin, co-trimaxazole, gentamycin, nalidixic acid, nitrofurantoin, and tetracycline was $90 \%, 0 \%, 20 \%, 30 \%, 25 \%, 70 \%, 10 \%, 40 \%, 50 \%, 5 \%, 85 \%, 25 \%$ respectively.

Multi-drug resistant organisms isolated in biofilm as shown in Table 3. There was a significant correlation between biofilm production and resistance to multiple antibiotics such as ciprofloxacin, nalidixic acid, cotrimoxazole, tetracycline 
Table 2. Antibiotic sensitivity pattern of organisms isolated in biofilm.

\begin{tabular}{|c|c|c|c|c|c|c|c|c|c|c|c|c|c|}
\hline \multirow[t]{2}{*}{ Organism Isolated } & \multicolumn{12}{|c|}{ Sensitive } & \multirow{2}{*}{$\begin{array}{c}\text { Resistant to all } \\
2 \text { (without } \\
\text { Amikacin 15) }\end{array}$} \\
\hline & $\mathrm{Ak}$ & $\mathrm{Am}$ & $\mathrm{Clx}$ & $\mathrm{Caz}$ & Cro & Chl & Cip & Sxt & $\mathrm{Gm}$ & $\mathrm{Nal}$ & Nif & $\mathrm{Te}$ & \\
\hline E. coli & $90 \%$ & 0 & $20 \%$ & $30 \%$ & $25 \%$ & $70 \%$ & $10 \%$ & $40 \%$ & $50 \%$ & $5 \%$ & $85 \%$ & $25 \%$ & \\
\hline Enterococcus faecalis & - & $53 \%$ & 0 & $\mathrm{R}-0$ & $\mathrm{~V}-0$ & - & - & - & $11.8 \%$ & - & - & 0 & \\
\hline Proteus mirabilis & $88 \%$ & 0 & $13 \%$ & $56 \%$ & $44 \%$ & 0 & $13 \%$ & 0 & $13 \%$ & 0 & $13 \%$ & 0 & \\
\hline Proteus vulgaris & $100 \%$ & 0 & $13 \%$ & $50 \%$ & $50 \%$ & 0 & $38 \%$ & 0 & $25 \%$ & 0 & $13 \%$ & 0 & \\
\hline Morganella morgani & $100 \%$ & 0 & 0 & 0 & $20 \%$ & 0 & 0 & $20 \%$ & 0 & 0 & $20 \%$ & 0 & \\
\hline Citrobacter diversus & $100 \%$ & 0 & 0 & $50 \%$ & $50 \%$ & 0 & $50 \%$ & 0 & $25 \%$ & 0 & $50 \%$ & 0 & \\
\hline Providencia retgeri & $75 \%$ & $25 \%$ & $25 \%$ & $50 \%$ & $75 \%$ & $25 \%$ & $50 \%$ & 0 & $25 \%$ & $25 \%$ & $50 \%$ & 0 & \\
\hline
\end{tabular}

Table 3. Multiple drug resistant pattern of biofilm organisms.

\begin{tabular}{ccc}
\hline Multiple Drug Combination & $\begin{array}{c}\text { Number of Isolates showing } \\
\text { Resistance }\end{array}$ & Percentage \\
\hline Tet, Sxt, Na, Cip, Amp & 43 & $42.15 \%$ \\
\hline
\end{tabular}

Tet $=$ Tetracycline, Sxt $=$ Co-trimoxazole, $\mathrm{Cip}=$ Ciprofloxacin, $\mathrm{Na}=$ Nalidixic acid, $\mathrm{Amp}=$ Ampicillin .

and ampicillin. Out of the 102 biofilm strains isolated, 43 (42.15\%) strains were multidrug resistant.

\section{Discussions}

95\% of the patients included in this study were asymptomatic since they are paralyzed patients from denden hospital, the other $5 \%$ of the catheterized patients had fever with chills and rigor. Similarly Tambyah et al. found more than $90 \%$ CAUTI were asymptomatic [2]. In this study $100 \%$ of both urine and biofilm samples showed growth of uropathogens and E. coli was the most frequently isolated pathogen in both samples, Figure 1 and Figure 2. A similar result was acquired by Majumder MI et al., their study showed $90 \%$ urine samples and $100 \%$ biofilm showed growth of uropathogens. E. coli was the most frequently isolated pathogen in both urine and biofilm ( $60 \%$ vs. $72 \%)$, followed by Klebsiella species (13\% vs $18 \%$ ) [7] [8]. Another study by Ronald showed that E. coli remains the predominant uropathogen $(80 \%)$ followed by $S$. saprophyticus $(10 \%$ - 15\%) [9]. Different study shows E. coli is responsible for more than $80 \%$ of symptomatic UTI and asymptomatic bacteriuria by expression of its variety of virulence factors and toxins [10].

Sensitivity pattern of $E$. coli in urine against amikacin, ampicillin, cephalexin, ceftazidime, ceftriaxone, chloramphenicol, ciprofloxacin, co-trimaxazole, gentamycin, nalidixic acid, nitrofurantoin, and tetracycline was $87.5 \%, 6.25 \%, 25 \%$. $31.25 \%, 43.75 \%, 87.5 \%, 25 \%, 31.25 \%, 56.25 \%, 18.75 \%, 81.25 \%, 31.25 \%$ respectively, when compared with the sensitivity pattern of $E$. coli in biofilm against amikacin, ampicillin, cephalexin, ceftazidime, ceftriaxone, chloramphenicol, ci- 
profloxacin, co-trimaxazole, gentamycin, nalidixic acid, nitrofurantoin, tetracycline was $90 \%, 0 \%, 20 \%, 30 \%, 25 \%, 70 \%, 10 \%, 40 \%, 50 \%, 5 \%, 85 \%, 25 \%$ respectively. We can simply observe in Table 1 and Table 2 that in most of the drugs resistance is frequently seen in biofilm isolates than in urine isolates except in three drugs. On a study done by JC Nickel et al., growth within the biofilm conferred a bacteria a resistance to tobramycin drug while the planktonic form of same bacteria was killed by tobramycin [11]. Since Amikacin is not prescribed as part of treatment for denden patients we can see that it has great sensitivity pattern that is because of the bacterial strains have not been introduced to this drug in vivo, that amikacin is not overprescribed and overused like the other 11 antibacterial drugs.

Antibiotic sensitivity patterns of biofilm and urine shows biofilm strains displayed relatively high resistance against majority of the tested antibiotics (9 out of 12 antibiotics showed high resistance against biofilm than urine). Highest sensitivity pattern was found for E. coli in urine for chloramphenicol (87.5\%) and lowest for ampicillin (6.25\%). Highest sensitivity pattern was found for $E$. coli in biofilm for nitrofurantoin (85\%) and lowest for ampicillin (0\%). Bacterial biofilms are often associated with long term persistence of organisms in various environments and they display an increased loss of sensitivity to antibiotics. According to Damien Balestrino et al., since biofilms are developing resistance to antibiotics, catheter lock solutions gave a promising idea to reduce biofilm formation [12]. There was a significant relation between biofilm formation and multidrug resistance in our study, see Table 3 , where $42.15 \%$ of organisms isolated from biofilm were multidrug resistant isolates, the five antibiotics used for investigating multidrug resistance of the biofilm organisms were from different drug families, ciprofloxacin from the fluoroquinolone, tetracycline from broad spectrum, cotrimaxazole which is trimethoprime, nalidixic acid from quinolones and ampicillin from B-lactam drugs. In the study done by Majumder MI et al. a similar finding was seen, where $68.69 \%$ of strains producing biofilm were multidrug resistant phenotypes [5].

In catheter biofilm resistant to all drugs were found in 15 samples where as in urine sample only 5 showed resistance to all drugs except amikacin, as amikacin is not over prescribed in denden hospital as other drugs we didn't use it for comparison, see rightmost column in Table 1 and Table 2.

\section{Recommendation}

- To establish a standard guideline for the indwelling urinary catheter management in Eritrea it requires a large-scale prospective study.

- Microbial biofilms respond poorly to conventional drug therapy as a result long-term infection of patients is inevitable, so we recommend that investigations to be carried out on how to eradicate biofilms.

- In order to reduce the economic load of CAUTI we have to increase our knowledge on how to use indwelling catheters safely. 
- Therapy against UTI should be guided by antimicrobial susceptibilities as urinary isolates are developing resistance to commonly used antibiotics.

- We strongly recommend the Ministry of health in Eritrea to implement studies done on eradication of biofilms.

\section{Conclusion}

In this study, a significant correlation between biofilm formation and multidrug resistance was observed. Since 15 samples from biofilm showed resistance to all tested antibiotics when compared to urine microorganisms showing only 5 of them resistant to all tested antibiotics and also since $42.15 \%$ of the biofilm strains showed multidrug resistance. $E$. coli was the most frequently isolated organism in both urine and biofilm samples.

\section{Conflicts of Interest}

The authors declare no conflicts of interest regarding the publication of this paper.

\section{References}

[1] Cunningham, A.B., Lennox, J.E. and Ross, R.J. (2001-2010) A Brief History of Biofilms. Eds. 2001-2010.

[2] Tambyah, P.A. and Maki, D.G. (2000) Catheter-Associated Urinary Tract Infection Is Rarely Symptomatic: A Prospective Study of 1497 Catheterized Patients. Archives of Internal Medicine, 160, 678-682.

[3] Clec'h, C., Schwebel, C., Français, A., Toledano, D., Fosse, J.P., et al. (2007) Does Catheter-Associated Urinary Tract Infection Increase Mortality in Critically Ill Patients. Infection Control \& Hospital Epidemiology, 28, 1367-1373.

[4] Allon, M. (2004) Dialysis Catheter-Related Bacteremia: Treatment and Prophylaxis. American Journal of Kidney.

[5] Stamm, W.E. (1991) Catheter-Associated Urinary Tract Infections: Epidemiology, Pathogenesis, and Prevention. The American Journal of Medicine, 91, 65S-71S. https://doi.org/10.1016/0002-9343(91)90345-X

[6] Costerton, J.W., Stewart, P.S. and Greenberg, E.P. (1999) Bacterial Biofilms: A Common Cause of Persistent Infections. Science, 284, 1318-1322. https://doi.org/10.1126/science.284.5418.1318

[7] Svanborg, C. and Godaly, G. (1997) Bacterial Virulence in Urinary Tract Infection. Infectious Disease Clinics of North America, 11, 513-529. https://doi.org/10.1016/S0891-5520(05)70371-8

[8] Majumder, M.I., et al. (2014) Bacteriology and Antibiotic Sensitivity Patterns of Urine and Biofilms in Patients with Indwelling Urinary Catheter in a Tertiary Hospital in Bangladish. Journal of Bacteriology and Parasitology, 5, 3. https://doi.org/10.4172/2155-9597.1000191

[9] Ronald, A. (2003) The Etiology of Urinary Tract Infection: Traditional and Emerging Pathogens.

[10] Ohkawa, M., Sugata, et al. (1990) Bacterial and Crystal Adherence to the Surfaces of Indwelling Urethral Catheters.

[11] Nickel, J.C., Ruseska, I., et al. (1985) Tobramycin Resistance of Pseudomonas aeru- 
ginosa Cells Growing as a Biofilm on Urinary Catheter Material.

https://doi.org/10.1128/AAC.27.4.619

[12] Balestrino, D., et al. (2009) Eradication of Microorganisms Embedded in Biofilm by an Ethanol-Based Catheter Lock Solution. Nephrology Dialysis Transplantation, 24. https://doi.org/10.1093/ndt/gfp187 